\title{
La recherche en obésité : apport des modèles animaux
}

\section{J. Dargent $\cdot$ S. Czernichow}

C) Springer-Verlag France 2012

Ce numéro particulièrement intéressant vous présente une série de deux articles sur le sucre.

Ces dossiers illustrent la complexité des relations entre la consommation de sucre ou produits sucrés et l'état de santé. Une tribune a aussi été ouverte aux professionnels du secteur qui donneront leur point de vue sur le sujet.

L'article de Dupuy et al. décrit une expérience originale de site internet dédié à l'obésité et la façon dont les patients, mais aussi les professionnels, le perçoivent ; alors que l'article d'Amro et al. fait une synthèse sur les liens épidémiologiques entre obésité et asthme.
Vous aurez aussi la chance de pouvoir découvrir une superbe revue sur la contribution apportée par les modèles animaux dans la compréhension des bases physiologiques et génétiques de l'obésité, en particulier sur les modèles de souris et rats. Enfin, sur le versant chirurgical, vous lirez avec intérêt une synthèse éclairée d'une sélection d'abstracts $\mathrm{du}$ XVIIe Congrès mondial de chirurgie bariatrique (IFSO) qui a eu lieu en Allemagne début septembre 2011.

Très bonne lecture à toutes et à tous... 